\title{
Genetic tools for highly pathogenic Francisella tularensis subsp. tularensis
}

Correspondence

Martin S. Pavelka, Jr

martin_pavelka@

urmc.rochester.edu

Received 6 May 2006

Revised 18 July 2006

Accepted 4 August 2006
Eric D. LoVullo,† Lani A. Sherrill, † Lanyn L. Perez $\ddagger$ and Martin S. Pavelka, Jr

University of Rochester Medical Center, 601 Elmwood Ave, Box 672, Rochester, NY 14642, USA

This paper is the first detailed description of the development and use of new genetic tools specifically for the safe manipulation of highly pathogenic Francisella tularensis subsp. tularensis. Most of these tools are also demonstrated to work with other F. tularensis subspecies. Kanamycin and hygromycin resistance determinants that function as genetic markers in F. tularensis subsp. tularensis strain Schu and sets of episomal shuttle vectors that are either unstable or stably maintained in the absence of selection were developed. In addition, the hyg gene, expressed from the F. tularensis groESL promoter, was successfully used as a marker for transposon mutagenesis. This work also includes the development of sacB-based suicide plasmids expressing kanamycin resistance that can be used for electroporation-mediated allelic exchange of unmarked mutations in Schu and the F. tularensis live vaccine strain (LVS). Using these plasmids, the two predicted $\beta$-lactamase genes, blaA and $b / a B$, in Schu and LVS were deleted. Only the $\Delta b / a B 1$ mutants had increased susceptibility to ampicillin, and this phenotype was complemented by a plasmid expressing $b / a B^{+}$. The results suggest that the $\beta$-lactam antibiotic resistance phenotype of Schu and LVS is likely due to only one of the two $\beta$-lactamase genes present and that ampicillin resistance can be used as an additional selectable marker in $\beta$-lactamase deletion mutants. The collection of tools presented in this report will be helpful for the genetic analyses of $F$. tularensis subsp. tularensis pathogenesis.

\section{INTRODUCTION}

Francisella tularensis is a fastidious Gram-negative bacterium responsible for the zoonotic disease tularaemia (Sjostedt et al., 1996). The disease has a variety of clinical presentations ranging from mild to more serious forms, such as pneumonic and typhoidal, which have a high mortality (Dennis et al., 2001). There are four subspecies of F. tularensis, each differing in their relative degree of pathogenesis in the human host, ranging from the weakly pathogenic subsp. novicida, the moderately pathogenic subspecies holarctica and mediasiatica, to the highly pathogenic subspecies tularensis (Garcia Del Blanco et al., 2002; Hollis et al., 1989; Johansson et al., 2001, 2004; Titball et al., 2003). The high mortality of serious forms of tularaemia, coupled with the very infectious nature of $F$. tularensis aerosols, prompted the past development of $F$. tularensis subsp. tularensis into a biological weapon (Dennis et al., 2001).

The biology and molecular pathogenesis of this organism have been difficult to study, primarily due to the need for

†These authors contributed equally to this work.

$\ddagger$ Present address: Biology Department, University of Maryland, Baltimore County, Baltimore, MD 21250, USA. biosafety level three conditions to manipulate the bacterium, its select agent status, and a lack of genetic tools. Most genetic studies have used the weakly pathogenic subspecies novicida or the live vaccine strain, LVS, derived from subsp. holarctica (Eigelsbach \& Downs, 1961; Golovliov et al., 2003; Lauriano et al., 2003; Nano et al., 2004; Sjostedt et al., 1996). So far, three Escherichia coli-Francisella shuttle plasmids have been described for use in these two subspecies (Maier et al., 2004; Norqvist et al., 1996; Pavlov et al., 1996); allelic exchange methods were developed for novicida and LVS (Golovliov et al., 2003; Lauriano et al., 2003), shuttle transposon mutagenesis has been used for novicida (Cowley et al., 2000), while the EZ::TN transposome system (Kawula et al., 2004) and a transposon based upon the Himarl element (Maier et al., 2006) have been used for mutagenesis of LVS. However, few genetic tools have been tested with highly pathogenic, select agent strains of $F$. tularensis subsp. tularensis. A recent report was the first to show that site-directed mutagenesis of strain Schu S4 is possible, using a conjugative $s a c B$ plasmid encoding chloramphenicol resistance (Twine et al., 2005).

A challenge in the development of genetic tools for Francisella is that only a few antibiotic resistance determinants can be used in highly pathogenic strains. All F. tularensis subspecies are intrinsically resistant to the 
$\beta$-lactam classes of antibiotics (Baker et al., 1985). Although most $F$. tularensis subspecies are sensitive to antibiotics such as streptomycin, gentamicin, tetracylines, chloramphenicol, kanamycin and erythromycin, except LVS, which is resistant to erythromycin (Dennis et al., 2001), the first four antibiotics can be used to treat tularaemia and thus the introduction of genes conferring resistance to these drugs into select agent strains of $F$. tularensis is restricted. The aminoglycosides kanamycin and nourseothricin are good antibiotics for genetic selection purposes in LVS but have not been tested in subsp. tularensis (Maier et al., 2004, 2006). Some of the currently available genetic tools encode tetracycline and/or chloramphenicol resistance, which limits their use to non-select agent strains of $F$. tularensis (Golovliov et al., 2003; Norqvist et al., 1996). Thus, there is a need to test marker genes and develop genetic tools for highly pathogenic strains of subsp. tularensis.

In this paper, we report the development of new E. coliFrancisella shuttle vectors and genetic tools for use in $F$. tularensis subsp. tularensis. We demonstrate that the use of the kanamycin resistance gene $a p h A-1$ as a selectable marker in strain Schu requires that the gene be cloned downstream of a F. tularensis promoter. We chose aphA-1 for this work, as it does not confer resistance to aminoglycosides used to treat F. tularensis infections (Dennis et al., 2001; Shaw et al., 1993). We also evaluated the hygromycin resistance gene, hyg, from Streptomyces hygroscopicus (Malpartida et al., 1983), as a selectable marker. We chose this antibiotic because it is stable, it is not used to treat $F$. tularensis infections (Dennis et al., 2001), and hyg does not confer resistance to other antibiotics (Leboul \& Davies, 1982; Malpartida et al., 1983). We also developed sacB-based suicide vectors and used them to delete the $\beta$-lactamase genes of strains Schu and LVS, and showed that efficient electroporation-mediated allelic exchange is possible in these organisms. Furthermore, we demonstrate that $\beta$ lactam resistance can be used as a selectable marker in these mutant strains.

\section{METHODS}

Bacterial strains, culture conditions and transformation. E. coli strain DH10B (Table 1) was used for routine cloning procedures and was grown in Luria-Bertani (LB) broth (BD Biosciences) or on $\mathrm{LB}$ agar. F. tularensis strains (Table 1) were grown at $37^{\circ} \mathrm{C}$ in liquid modified Mueller-Hinton medium (MMH), which is MuellerHinton broth (BD Biosciences) supplemented with $1 \mathrm{mM} \mathrm{CaCl}$, $1 \mathrm{mM} \mathrm{MgCl}, 0 \cdot 1 \%(\mathrm{w} / \mathrm{v})$ glucose, $0 \cdot 025 \%(\mathrm{w} / \mathrm{v})$ ferric pyrophosphate and $2 \cdot 0 \%(\mathrm{v} / \mathrm{v})$ IsoVitaleX (BD Biosciences), or on $\mathrm{MMH}$ agar, which is the MMH medium above supplemented with $1.0 \%$ $(\mathrm{w} / \mathrm{v})$ proteose peptone (BD Biosciences), $2 \cdot 5 \%(\mathrm{v} / \mathrm{v})$ defibrinated sheep blood (Remel) and $1.5 \%(\mathrm{w} / \mathrm{v})$ bacto-agar (BD Biosciences) but lacking additional $\mathrm{CaCl}_{2}$ and $\mathrm{MgCl}_{2}$. Broth cultures of F. tularensis strains Utah 112 and LVS were grown in glass culture flasks with slow shaking (100 r.p.m.), while small $(<20 \mathrm{ml})$ liquid cultures of $F$. tularensis strain Schu were grown in $30 \mathrm{ml}$ plastic medium bottles with slow shaking and larger cultures of 50-100 ml were grown in $450 \mathrm{~cm}^{2}$ plastic roller bottles (Corning) incubated on a roller apparatus. F. tularensis agar cultures were typically incubated for $48 \mathrm{~h}$. When necessary, ampicillin (Sigma-Aldrich Chemical) was added at 100 or $50 \mu \mathrm{g} \mathrm{ml}^{-1}$, respectively, for E. coli or F. tularensis, while kanamycin (Sigma-Aldrich) was used at $50 \mu \mathrm{g} \mathrm{ml}^{-1}$ for $E$. coli, $5 \mu \mathrm{g} \mathrm{ml}^{-1}$ for LVS and Schu, or $15 \mu \mathrm{g} \mathrm{ml}^{-1}$ for Utah 112 . Kanamycin stock solutions were made by accounting for the concentration of active kanamycin reported for each lot by the supplier. Hygromycin B (Roche Applied Science) was used at $200 \mu \mathrm{g} \mathrm{ml}^{-1}$ for all species and strains. Sucrose was used at a final concentration of $8 \%(\mathrm{w} / \mathrm{v})$. EZ:: TN transposomes (Epicentre Technologies) were constructed as recommended by the manufacturer and stored at $-20{ }^{\circ} \mathrm{C}$ for at least 3 days prior to use.

Electroporations were done with $F$. tularensis cells grown in $\mathrm{MMH}$ broth to an $\mathrm{OD}_{600}$ of $0 \cdot 2-0 \cdot 5$ for strain Schu, and $0 \cdot 5-1 \cdot 0$ for Utah 112 and LVS. The cells were pelleted, washed twice in sterile, room temperature $0.5 \mathrm{M}$ sucrose, and resuspended in sucrose at $1 / 100$ of the original culture volume. Approximately $0 \cdot 1-1 \cdot 0 \mu \mathrm{g}$ plasmid DNA was used for each electroporation mixed together with $100 \mu$ prepared cells in a $0 \cdot 2 \mathrm{~cm}$ gap cuvette. For allelic exchange experiments, we used at least $1 \mu \mathrm{g}$ Qiagen column-prepared suicide plasmid per electroporation. The electroporations were done at room temperature with a BioRad Gene Pulser II set for $2.5 \mathrm{kV}, 25 \mu \mathrm{F}$ and $600 \Omega$, followed by the addition of $1 \mathrm{ml}$ room temperature $\mathrm{MMH}$ broth to the cuvette, transfer of the cell mixture to a $15 \mathrm{ml}$ conical tube, and incubation with shaking at $37^{\circ} \mathrm{C}$ for $3-4 \mathrm{~h}$. Transformants were selected on $\mathrm{MMH}$ plates supplemented with the appropriate antibiotics.

DNA manipulation. DNA methods were performed essentially as described previously (Ausubel et al., 1987). DNA fragments were isolated using agarose gel electrophoresis and absorption to a silica matrix (GeneClean; Bio 101), or by QIAquick spin columns (Qiagen). Oligonucleotides were synthesized by Invitrogen Life Technologies. All restriction endonucleases and DNA-modifying or polymerase enzymes were from New England Biolabs, Fermentas or Roche. PCR reactions with various thermostable polymerases were done according to the manufacturer's recommendations. All plasmids used in this study (Table 1) were from our laboratory's collection, except the cryptic Francisella plasmid pFNL10 (Pomerantsev et al., 2001a), which was a generous gift from Fran Nano of the University of Victoria, BC, Canada, and the shuttle vector pFNLTP1 (Maier et al., 2004), which was kindly provided by Tom Zahrt of the Medical College of Wisconsin. Plasmids were prepared from E. coli and $F$. tularensis strains using either a standard alkaline lysis protocol or with Qiagen columns. Genomic DNA from F. tularensis was prepared by a rapid guanidinium thiocyanate method previously described for mycobacteria (Pavelka \& Jacobs, 1999).

Plasmid construction. Detailed descriptions of the construction of the plasmids used in this study can be obtained from the corresponding author. Information pertinent to the understanding of this work is described below for some of the plasmids.

Shuttle vector pMP393. Plasmid pMV261 (Table 1) was the source of a $2 \cdot 1 \mathrm{~kb}$ HincII-MluI DNA fragment containing the ColE1 origin of replication and the $a p h A-1$ gene. This fragment was ligated to a $2 \cdot 0 \mathrm{~kb}$ DNA fragment obtained from pFNL10 via PCR that encompasses the region from position 1 through 2000 of the pFNL10 GenBank sequence (accession no. NC_004952) to produce pMP393.

Shuttle vectors pMP527 and pMP529. The aphA-1 gene was obtained from pMV261 using PCR to generate an NdeI site at the start codon of the gene. This fragment was cloned into pMP478, which is pMV261 bearing a PCR-generated NotI-NdeI DNA fragment from LVS (GenBank accession no. AM23362) corresponding to $290 \mathrm{bp}$ upstream of the groESL operon, encompassing the promoter 
Table 1. Bacterial strains and plasmids

\begin{tabular}{|c|c|c|}
\hline Strains or plasmid & Description & Source or reference ${ }^{\star}$ \\
\hline \multicolumn{3}{|l|}{ Bacteria } \\
\hline \multicolumn{3}{|l|}{ E. coli } \\
\hline DH10B & 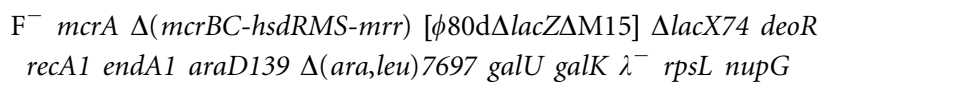 & \\
\hline \multicolumn{3}{|l|}{ F. tularensis } \\
\hline Utah 112 & F. tularensis subsp. novicida & K. Elkins \\
\hline LVS & F. tularensis subsp. holarctica live vaccine strain & J. Benach \\
\hline Schu & F. tularensis subsp. tularensis & M. Schriefer \\
\hline PM1521 & LVS $\triangle b l a A 1$ & This work \\
\hline PM1556 & LVS $\Delta$ blaB1 & This work \\
\hline PM1560 & LVS $\triangle$ blaA1 $\triangle b l a B 1$ & This work \\
\hline PM1516 & Schu $\triangle b l a A 1$ & This work \\
\hline PM1580 & Schu $\Delta b l a B 1$ & This work \\
\hline PM1571 & Schu $\Delta b l a A 1 \Delta b l a B 1$ & This work \\
\hline \multicolumn{3}{|l|}{ Plasmids } \\
\hline pMOD-2 & $\mathrm{Ap}^{\mathrm{R}}, \mathrm{EZ}: \mathrm{:TN}$ cloning vector & Epicentre \\
\hline pFNL10 & Francisella cryptic plasmid & Pomerantsev et al. (2001a) \\
\hline pFNLTP1 & $\mathrm{Km}^{\mathrm{R}}$, E. coli-F. tularensis shuttle vector & Maier et al. (2004) \\
\hline pMV261 & $\mathrm{Km}^{\mathrm{R}}$, E. coli-mycobacterial shuttle vector & Stover et al. (1991) \\
\hline pMV261.hyg & $\mathrm{Hyg}^{\mathrm{R}}$, E. coli-mycobacterial shuttle vector & Flores et al. (2005b) \\
\hline pYUB631 & $\mathrm{Km}^{\mathrm{R}}$, pMV261 bearing the $s a c R$ sacB region of Bacillus subtilis & Pavelka \& Jacobs (1999) \\
\hline pMP393 & $\mathrm{Km}^{\mathrm{R}}$, E. coli-F. tularensis shuttle vector & This work \\
\hline pMP478 & $\begin{array}{l}\mathrm{Km}^{\mathrm{R}} \text {, pMV261 bearing a } 290 \mathrm{bp} X b a \mathrm{I}-\mathrm{NdeI} \text { fragment containing the } \\
\text { groESL promoter of LVS }\end{array}$ & This work \\
\hline pMP492 & $\begin{array}{l}\mathrm{Km}^{\mathrm{R}} \text {, pMV261 bearing a } 1.8 \mathrm{~kb} \text { Pst } \mathrm{PCR} \text { fragment containing the blaB }{ }^{+} \\
\text {region from LVS }\end{array}$ & This work \\
\hline pMP521 & $\begin{array}{l}\mathrm{Km}^{\mathrm{R}} \text {, pMV261 bearing a } 3.2 \mathrm{~kb} \text { PstI PCR fragment containing the blaA }{ }^{+} \\
\text {region from LVS }\end{array}$ & This work \\
\hline pMP523 & $\begin{array}{l}\mathrm{Km}^{\mathrm{R}} \text {, pMP478 bearing the aphA-1 gene from pMV261 cloned under } \\
\text { control of } P_{\text {groESL }}\end{array}$ & This work \\
\hline pMP524 & $\begin{array}{l}\text { Hyg }^{\mathrm{R}} \text {, pMP478 bearing the hyg gene from pMV261.hyg, cloned under } \\
\text { control of } P_{\text {groESL }}\end{array}$ & This work \\
\hline pMP527 & $\mathrm{Km}^{\mathrm{R}}$, E. coli-F. tularensis shuttle vector with the $P_{\text {groESL}}-a p h A-1$ cassette & This work \\
\hline pMP528 & $\mathrm{Ap}^{\mathrm{R}}, \mathrm{Hyg}^{\mathrm{R}}, \mathrm{pMOD}-2$ bearing the $P_{\text {groESL }}-h y g$ cassette & This work \\
\hline pMP529 & Hyg $^{\mathrm{R}}$, E. coli-F. tularensis shuttle vector with the $P_{\text {groESL }}$-hyg cassette & This work \\
\hline pMP537 & $\begin{array}{l}\mathrm{Km}^{\mathrm{R}} \text {, deletion derivative of pMP492 made by inverse PCR resulting in } \\
\text { the } \Delta \text { blaB1 allele }\end{array}$ & This work \\
\hline pMP552 & $\begin{array}{l}\mathrm{Km}^{\mathrm{R}} \text {, pLLP18 bearing a } 1 \cdot 3 \mathrm{~kb} \text { fragment that is the } 3^{\prime} \text { partial } \Delta \text { blaA } 1 \text { allele, } \\
\text { forming the complete deletion allele }\end{array}$ & This work \\
\hline pMP578 & $\mathrm{Km}^{\mathrm{R}}$, first-generation $F$. tularensis sacB suicide vector & This work \\
\hline pMP582 & $\begin{array}{l}\mathrm{Km}^{\mathrm{R}} \text {, pMP578 suicide vector bearing the } 2 \cdot 2 \mathrm{~kb} \text { fragment containing the } \\
\Delta \text { blaA1 allele from pMP522 }\end{array}$ & This work \\
\hline pMP590 & $\begin{array}{l}\mathrm{Km}^{\mathrm{R}}, 570 \text { bp smaller, second-generation } F \text {. tularensis sacB suicide vector } \\
\text { derived from pMP588 }\end{array}$ & This work \\
\hline pMP592 & $\mathrm{Km}^{\mathrm{R}}$, pMP590 suicide vector bearing the $\Delta$ blaB1 allele from pMP537 & This work \\
\hline pMP607 & $\begin{array}{l}\mathrm{Km}^{\mathrm{R}} \text {, E. coli-F. tularensis shuttle vector with the } P_{\text {groESL}}-a p h A-1 \text { cassette } \\
\text { and ORF4-ORF5 of pFNL10 }\end{array}$ & This work \\
\hline pMP615 & $\begin{array}{l}\mathrm{Hyg}^{\mathrm{R}} \text {, pMP529 with a } 1.4 \mathrm{~kb} M l u \mathrm{I} \text { PCR fragment bearing the } b l a B^{+} \\
\text {gene of Schu }\end{array}$ & This work \\
\hline pMP633 & $\begin{array}{l}\text { Hyg }{ }^{\mathrm{R}} \text {, E. coli-F. tularensis shuttle vector with the } P_{\text {groESL }} \text {-hyg cassette and } \\
\text { ORF4-ORF5 of pFNL10 }\end{array}$ & This work \\
\hline
\end{tabular}

${ }^{\star}$ K. Elkins, FDA, Rockville, MD, USA; J. Benach, SUNY, Stony Brook, NY, USA; M. Schriefer, CDC, Fort Collins, CO, USA. 
and the groES start codon, engineered to have the NdeI site at the ATG. The resultant plasmid, pMP523 (Table 1), was used as the source of the $P_{\text {groESL}}-a p h A-1$ cassette for the construction of pMP527.

The hyg gene was amplified from pMV261.hyg using PCR to produce a $1012 \mathrm{bp} h y g$ product with an engineered NdeI site at the start codon of the gene, which was cloned into pMP478 as described above for the aphA-1 cassette, yielding pMP524. The $P_{\text {groESL }}$-hyg cassette was cloned into pMP393 to generate pMP529 in the same manner as the $P_{\text {groESL- }}$ aphA-1 cassette was cloned above in the construction of pMP527.

Shuttle vectors pMP607 and pMP633. pMP607 was derived from pMP527 and contains an ORF4-ORF5 DNA fragment (encompassing nucleotide positions 789-2596 of the pFNL10 sequence) obtained by PCR. pMP633 was made by swapping the $P_{\text {groESL }}-a p h A-1$ cassette of pMP607 with the $P_{\text {groESL }}-h y g$ cassette from pMP529.

sacB suicide vectors. Plasmid pMP578 was constructed by ligating a $2.3 \mathrm{~kb} B g l \mathrm{II}-E c o \mathrm{RV}$ DNA fragment bearing the $s a c B$ region from pYUB631 (Table 1) with a $3 \cdot 2 \mathrm{~kb}$ BglII-HincII DNA fragment from pMP527. pMP57 lacks ORF2 and part of repA of the Francisella origin of replication. Plasmid pMP590 is a modified version of pMP578 that includes a multiple cloning site and two deletions that move $s a c B$ closer to the repA promoter region.

Plasmid for $\Delta b / a A$ allelic exchange, pMP582. A DNA fragment containing blaA was obtained from LVS genomic DNA using PCR and cloned into pMV261 to yield pMP521. An in-frame deletion of $771 \mathrm{bp}$ within blaA was made using PCR. A $2 \cdot 1 \mathrm{~kb}$ fragment con- taining the $\triangle b l a A 1$ allele was cloned into pMP578 to yield pMP582. There is $1076 \mathrm{bp}$ of DNA upstream and $1120 \mathrm{bp}$ downstream of the $\triangle b l a A 1$ allele in this plasmid.

Plasmid for $\triangle b l a B$ allelic exchange, pMP592. A DNA fragment containing blaB was obtained from LVS genomic DNA using the PCR and cloned into pMV261 to yield pMP492, which was used as a template for inverse PCR to generate an in-frame deletion of $735 \mathrm{bp}$ within blaB. This allele was cloned into the EcoRV site within pMP590 to produce pMP592. There is $485 \mathrm{bp}$ of DNA upstream and $399 \mathrm{bp}$ downstream of the $\Delta$ blaB1 allele in this plasmid.

Plasmid for blaB complementation, pMP615. The $b l a B^{+}$gene with its putative promoter region was obtained from Schu genomic DNA (GenBank accession no. AJ749949) as a $1.4 \mathrm{~kb}$ DNA fragment with engineered $M l u \mathrm{I}$ sites using PCR, and cloned into the MluI site of pMP529 to yield pMP615.

\section{RESULTS}

\section{First-generation shuttle vectors}

Our backbone E. coli-Francisella shuttle vector, pMP393 (Fig. 1), is derived from plasmid pMV261, an E. colimycobacterial shuttle vector (Stover et al., 1991). We replaced the mycobacterial pAL5000 origin of replication in pMV261 with a PCR-generated, $2 \mathrm{~kb}$ DNA fragment
A.

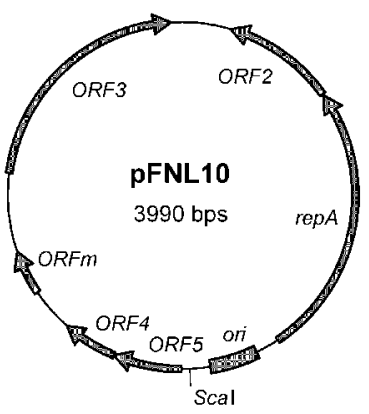

D.

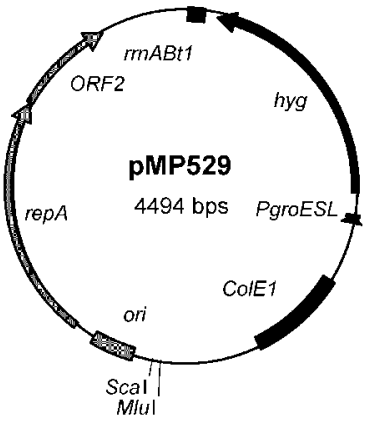

B.

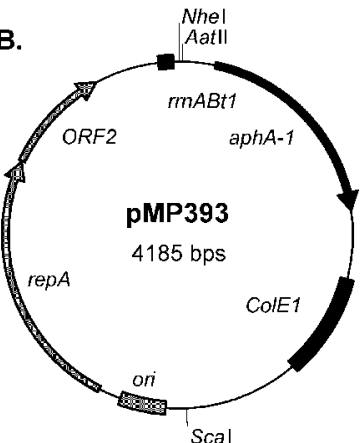

E.

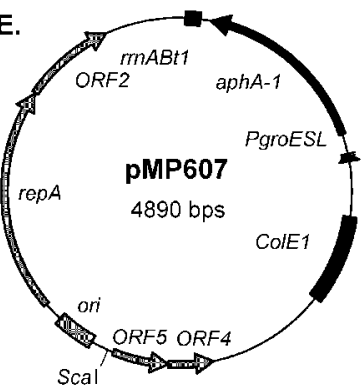

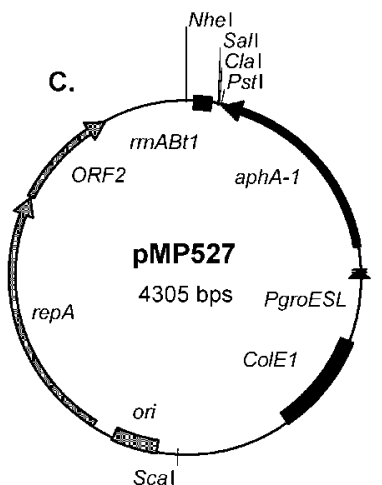

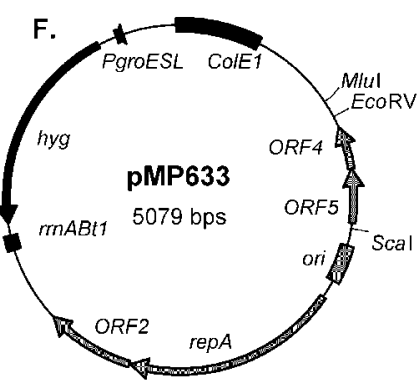

Fig. 1. Maps of E. coli-F. tularensis shuttle vectors. (A) The F. tularensis cryptic plasmid pFNL10 is shown for comparison. The plasmid pMP393 (B) contains the Francisella origin of replication region (ori, repA, ORF2) from pFNL10 and the E. coli ColE1 origin of replication, gene aphA-1 (from Tn903) conferring resistance to kanamycin, and the rrnABt1 transcriptional

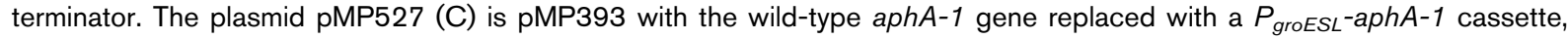
while pMP529 (D) is essentially the same as pMP527 but contains the $P_{\text {groEsL }}$-hyg cassette. The ORF4-ORF5-containing plasmid, pMP607 (E), was derived from pMP527, and pMP633 (F) was derived from pMP607. 
from the Francisella cryptic plasmid pFNL10 (Fig. 1) (Pomerantsev et al., 2001a) that includes the origin of replication, the repA and ORF2 genes and their promoter region, but no other intact ORFs from pFNL10.

We routinely obtained $\sim 10^{6}$ kanamycin-resistant LVS transformants per electroporation with saturating amounts of pMP393 DNA (Fig. 2). Calculating our transformation efficiency on a per $\mu \mathrm{g}$ basis yields a value of $\sim 10^{7}$ kanamycin-resistant transformants ( $\mu$ g plasmid DNA) ${ }^{-1}$, which is the same as that previously reported by others for plasmid transformation of LVS following electroporation (Maier et al., 2004).

We found that the transformation efficiencies of strains Utah 112 and Schu were three to four orders of magnitude lower than that of LVS (Fig. 2). The low transformation efficiency of Utah 112 we obtained has been reported by others and is likely due to a restriction-modification system previously described in this strain (Maier et al., 2004). We hypothesized that the aphA-1 gene in pMP393 might not be expressed well in Schu, and so we replaced the wild-type aphA-1 in pMP393 with a PCR-generated cassette consisting of aphA-1 expressed from the F. tularensis groESL promoter (Ericsson et al., 1997) to produce pMP527 (Fig. 1). With this plasmid we obtained transformation efficiencies of at least $10^{6}$ with both LVS and Schu, and a $10^{3}$ transformation efficiency with Utah 112 (Fig. 2). Transformants of all three strains that received pMP393 or pMP527 grew at the same rate and had normal colony morphologies (data not shown).

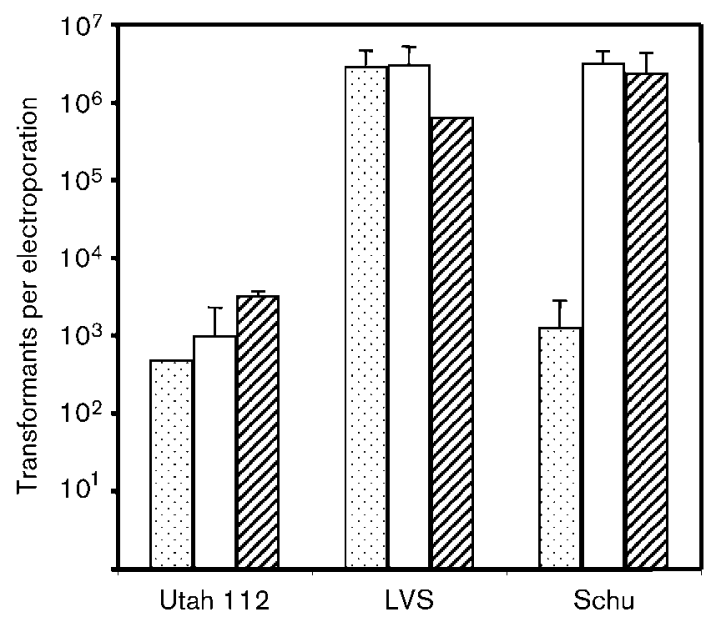

Fig. 2. Transformation efficiencies of shuttle vectors with the three $F$. tularensis subspecies strains. The efficiencies are expressed as the number of antibiotic-resistant colony-forming units per electroporation with saturating amounts of plasmid DNA. Shown are the mean values and standard deviations of multiple experiments. Key: pMP393 (aphA-1, stippled bars),

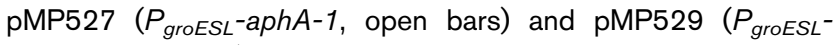
hyg, hatched bars).
We compared transformation efficiencies of strains LVS and Schu with pMP393, pMP527 and the previously developed E. coli-Francisella shuttle vector pFNLTP1 capable of highly efficient transformation of LVS (Maier et al., 2004) (Table 1). This vector consists of the E. coli pCR2.1-TOPO cloning vector fused to most of the pFNL10 cryptic plasmid. It differs from pMP393 and pMP527 in that it has ORF3, part of ORF5 and the npt gene for neomycin/ kanamycin resistance, but it has ori, repA and ORF2 as they are present in our vectors. We found that the pFNLTP1 transformation efficiency was very high with strain LVS, as expected, but low with strain Schu. We obtained $\sim 10^{6}$ transformants with LVS and less than 50 transformants with strain Schu per electroporation (data not shown). The electroporations with pFNLTP1 were done in parallel with the cells and media used for the experiments with pMP393 and pMP527 shown in Fig. 2. The Schu/pFNLTP1 transformants took 2-3 days longer to grow than pMP393 or pMP527 transformants and had varying colony morphology and size, ranging from small pinpoints to an almost normal size. However, in agreement with a previous report (Maier et al., 2004), we saw no growth or morphological changes for the LVS/pFNLTP1 transformants.

\section{Hygromycin resistance as a selectable marker}

We developed the hyg gene, encoding a hygromycin phosphotransferase, into a selectable marker by building a new derivative plasmid, pMP529, made by replacing aphA-1 of pMP393 with a PCR-generated cassette bearing hyg cloned downstream of the F. tularensis groESL promoter as was done for the aphA-1 cassette above (Fig. 1). We obtained essentially the same transformation efficiencies with pMP529 as we did with pMP527 and the three $F$. tularensis subspecies, obtaining $\sim 10^{6}$ transformants per electroporation with strains LVS and Schu, and $\sim 10^{3}$ hygromycin-resistant clones per electroporation with strain Utah 112 (Fig. 2). We used hygromycin at a concentration of $200 \mu \mathrm{g} \mathrm{ml}^{-1}$ for these experiments because this concentration is sufficient for selection against non-transformed $F$. tularensis. Furthermore, the frequency of spontaneous resistance of $F$. tularensis strains to the antibiotic was $<10^{-8}$ when used at this concentration (data not shown).

\section{Plasmid maintenance and second-generation shuttle vectors}

We investigated the maintenance of the pMP527 shuttle vector in strains LVS and Schu in the absence of antibiotic selection and found that the plasmid was lost after subculture in media lacking kanamycin. For this determination, overnight cultures of pMP527 transformants grown in kanamycin medium were subcultured 1:10 into fresh medium with or without kanamycin and incubated for an additional $24 \mathrm{~h}$, after which time we plated cells onto solid medium lacking antibiotic. Colonies were then picked and patched onto medium with or without kanamycin. The 
percentage of kanamycin-sensitive clones in each population is shown in Table 2.

We hypothesized that introducing additional DNA from the cryptic plasmid pFNL10 into our first-generation shuttle vectors might allow us to develop a second-generation series of vectors that are stably maintained in a population grown without antibiotic selection. To this end, we cloned the ORF4-ORF5 region of pFNL10, encoding a putative 'addiction system' (Pomerantsev et al., 2001a), into pMP527 to produce pMP607 (Fig. 1). The cloning was designed to reconstruct the relevant region in pMP607 as it organized in pFNL10 (Fig. 1).

Electroporation experiments with pMP607 and strains LVS and Schu revealed no defect in transformation efficiency as compared to the parental plasmid pMP527 (data not shown). When we repeated the stability tests, we found that pMP607 was effectively maintained in populations of transformed strains in the absence of selection. At most, only $2 \%$ of the population was kanamycin sensitive after $24 \mathrm{~h}$ growth in broth lacking the antibiotic (Table 2). Continued subculturing of LVS/pMP607 transformants for 2 days in the absence of antibiotic selection did not increase the percentage of kanamycin-sensitive cells (data not shown).

To improve the utility of the vector pMP607, we constructed a derivative, pMP633 (Fig. 1), which has the $P_{\text {groESL-aphA-1 }}$ cassette replaced with the $P_{\text {groESL }}-h y g$ cassette. This plasmid has high transformation efficiency like its parent vector and is also maintained in a population of transformants without selection (Table 2).

\section{Transposon mutagenesis}

We tested the ability of the $P_{\text {groESL }}-h y g$ cassette to function at single copy in the F. tularensis chromosome by incorporating the cassette into a transposon and using it to mutagenize F. tularensis strains LVS and Schu. We used the commercially available EZ: :TN transposome system from Epicentre Technologies, which was previously shown to work well for mutagenesis of LVS (Kawula et al., 2004). The transposome consists of a purified transposase enzyme bound to the EZ::TN transposon, a hyperactive version of

Table 2. Maintenance of shuttle vectors

\begin{tabular}{|c|c|c|}
\hline \multirow[t]{2}{*}{ Plasmid } & \multicolumn{2}{|c|}{ Percentage loss of antibiotic resistance ${ }^{\star}$} \\
\hline & LVS & Schu \\
\hline pMP527 & $74,98(100)$ & $65,59,70(100)$ \\
\hline pMP607 & $0,2(50)$ & $2,0,0(50)$ \\
\hline pMP633 & $0,0(50)$ & Not determined \\
\hline
\end{tabular}

${ }^{\star}$ The percentage of screened clones that lost antibiotic resistance is shown with the number of clones screened in each experiment in parentheses. Two cultures were tested for each plasmid in LVS and three cultures tested for each plasmid in strain Schu. the transposon Tn 5 characterized by two 19 bp mosaic ends (ME) flanking an antibiotic resistance marker (Goryshin et al., 2000). A complex formed in vitro by the transposase and transposon DNA is stable in the absence of magnesium and can be electroporated into bacteria, where intracellular magnesium ions initiate transposition (Goryshin et al., 2000).

We constructed a new transposon by cloning the $P_{\text {groEsL }}-h y g$ cassette into the pMOD-2 vector from Epicentre, which contains the mosaic ends of the EZ::TN transposon flanking a multiple cloning site, to produce the plasmid pMP528 (Fig. 3). Cleavage of the plasmid with the restriction enzyme $P v u I I$ releases transposon DNA fragments which are purified and complexed with the EZ::TN transposase.

We electroporated transposomes into strains LVS and Schu and selected for insertional mutants on $\mathrm{MMH}$ medium supplemented with $200 \mu \mathrm{g}$ hygromycin $\mathrm{ml}^{-1}$. For both strains we obtained hygromycin-resistant transformants at a frequency of $\sim 10^{-6}$ per viable colony-forming unit. Three independent electroporations with LVS yielded $0.6 \times 10^{3}$, $1 \cdot 3 \times 10^{3}$ and $1.6 \times 10^{3}$ hygromycin-resistant mutants per electroporation while two electroporations of two independent cultures of Schu yielded $2 \cdot 2 \times 10^{3}, 2 \cdot 2 \times 10^{3}, 2 \cdot 5 \times 10^{3}$ and $2 \cdot 6 \times 10^{3}$ hygromycin-resistant mutants per electroporation. We did not obtain hygromycin-resistant clones in electroporations lacking transposomes. We performed Southern blots on genomic DNA prepared from ten arbitrarily chosen hygromycin-resistant transposon mutants of Schu using the hyg gene as the probe. Of the ten mutants, one had two insertions, while the other nine appeared to have only one transposon insertion each, which was located at a different site in each chromosome relative to the other mutants (Fig. 3). This frequency of multiple insertions was previously described by us, and others, who have used the EZ:: TN system in mycobacteria, and is likely the result of a single cell receiving two or more transposomes during the electroporation (Derbyshire et al., 2000; Flores et al., 2005a).

\section{Suicide plasmids and allelic exchange of bla genes}

We used the first-generation shuttle vector pMP527 (Fig. 1) as the backbone plasmid to construct a $s a c B$-based allelic exchange vector. Our first $s a c B$ vector, pMP578 (Fig. 4), was constructed by deleting part of repA and all of the ORF2 gene of the Francisella origin of replication of pMP527 (Fig. 1) and then inserting a fragment bearing the $s a c B$ region into the backbone of the plasmid. This was done to ensure that the vector would not replicate in Francisella and that $s a c B$ would be expressed from a strong Francisella promoter, presumably that which originally drove transcription of repA and ORF2.

We tested the ability of this plasmid to delete the $\beta$ lactamase genes in LVS and Schu using a two-step allelic 
A.

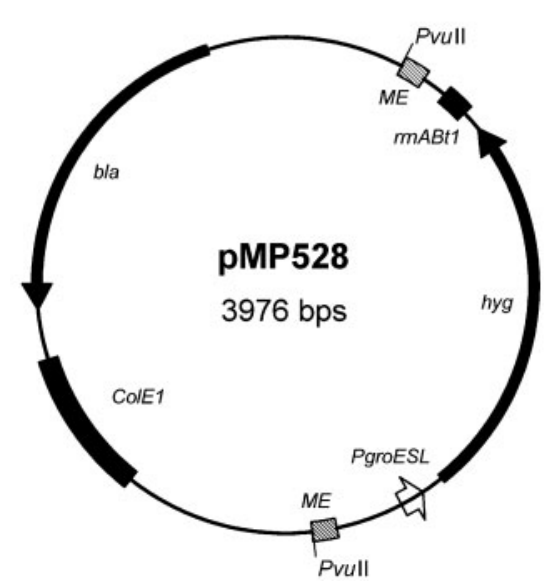

B.
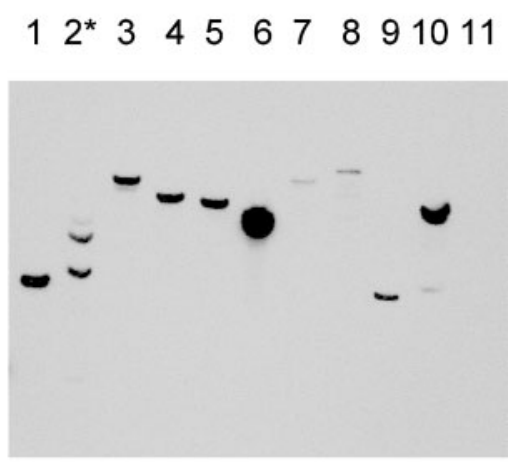

Fig. 3. $E Z:: T N$ plasmid and Southern blot of Schu mutants. (A) Map of the pMP528 plasmid, a derivative of the EZ::TN

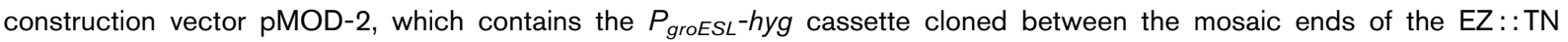
transposon. The $1.6 \mathrm{~kb}$ transposon is removed from pMP528 by digestion with the restriction endonuclease $P v u l l$. (B) Southern blot of Pvull-digested genomic DNA from Schu transposon mutants and wild-type, probed with hyg. Lanes: 1-10, transposon mutants; 11, wild-type Schu. The asterisk indicates the double insertion mutant (lane 2). Overexposure of the blot did not reveal any additional bands in lanes 7 and 8.

exchange. Previous work by another group showed that disruption of bla (designated blaA here), encoding a putative class A $\beta$-lactamase, does not affect the resistance phenotype of subsp. novicida strain Utah 112 and revealed that a second, putative class A $\beta$-lactamase gene (designated blaB here) may contribute to resistance (Lauriano et al.,
2003). We used the $s a c B$ vector pMP578 to construct pMP582, an allelic exchange plasmid for $\triangle b l a A 1$, and a second-generation $s a c B$ vector, pMP590 (Fig. 4), that is smaller than pMP578 and has multiple cloning sites, and used it to make the $\triangle b l a B 1$ allelic exchange plasmid pMP592.

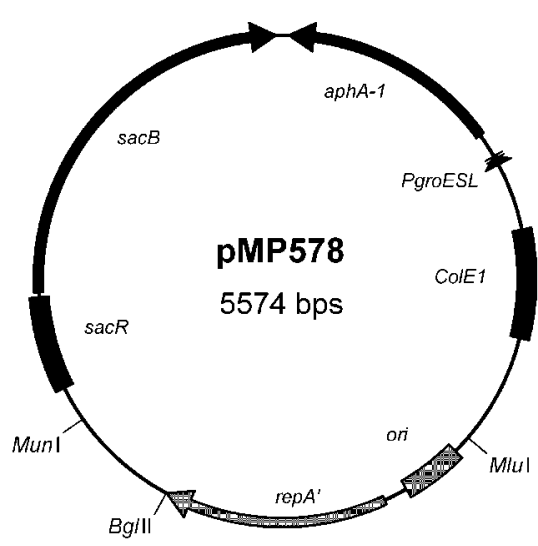

A.

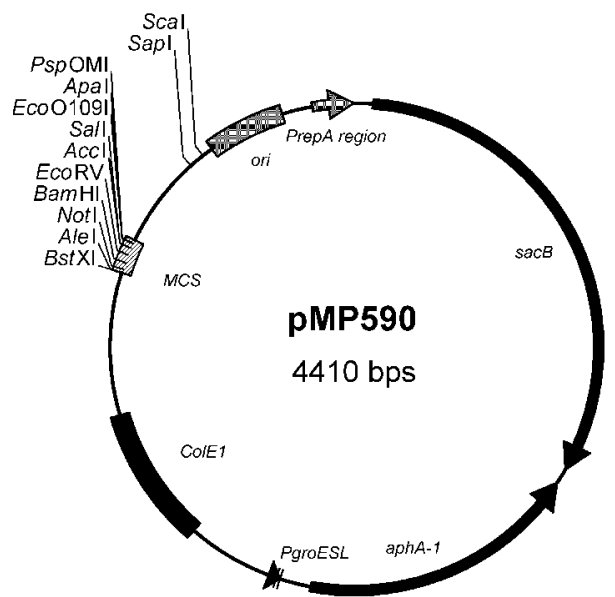

B.

Fig. 4. Maps of the F. tularensis sacB suicide vectors pMP578 and pMP590. The plasmid pMP578 (A) is derived from pMP527 and is missing ORF2 and part of $\operatorname{repA}$. It also contains $\operatorname{sac} B$ and the regulatory region sacR. The plasmid pMP590 $(B)$ is a $1.1 \mathrm{~kb}$ smaller version of pMP578 containing a multiple cloning site (MCS). The deletion in pMP590 removes the $s a c R$ region and moves the $\operatorname{sac} B$ gene closer to the repA promoter region. Both plasmids confer kanamycin resistance to Schu and LVS. 
A typical allelic exchange procedure was done by electroporating the suicide vectors into LVS or Schu and plating on medium containing kanamycin at $5 \mu \mathrm{g} \mathrm{ml}^{-1}$. Each suicide plasmid yielded kanamycin-resistant recombinants at a frequency of $10^{-5}$ to $10^{-6}$ relative to the transformation efficiency $\left(\sim 10^{6}\right.$ transformants per electroporation) with the replicating plasmid pMP527. Electroporations using suicide vectors lacking a $F$. tularensis DNA insert did not yield kanamycin-resistant recombinants. All the kanamycinresistant primary recombinants arising from the $\triangle b l a A 1$ and $\triangle$ blaB2 suicide plasmid transformations were sucrose sensitive, which was presumed to reflect integration of the suicide plasmid into the native locus of the chromosome by a single homologous recombination event.

We grew kanamycin-resistant, sucrose-sensitive, primary recombinants to saturation in medium lacking antibiotic and then plated the cells onto sucrose medium to select against clones that did not undergo a second recombination event. For all our experiments, sucrose-resistant clones arose from these cultures at frequencies of $10^{-3}$ to $10^{-4}$ relative to the viable counts. These putative secondary recombinants were then screened for loss of kanamycin resistance as an indication of successful recombination and loss of the integrated suicide plasmid. In the secondary recombinant pool, all the sucrose-resistant clones should be kanamycin sensitive, and in general, the population will consist of both wild-type and mutant clones. In practice, however, one always finds a few kanamycin-resistant clones in the sucroseresistant population that represent clones that did not undergo a secondary recombination event but, instead, have a mutation (possibly an insertion element or deletion) within the $\operatorname{sac} B$ gene or promoter region. In our experiments, we typically found kanamycin resistance in the sucrose-resistant population at frequencies ranging from 0 to $30 \%$.

We screened a set of sucrose-resistant, kanamycin-sensitive secondary recombinants by PCR to identify the $\triangle$ blaA1 mutants. We picked two mutants, PM1521 (LVS $\Delta$ blaA1) and PM1516 (Schu $\Delta$ blaA1), for subsequent work. A Southern blot showing the deletion of blaA in these two strains is shown in Fig. 5(A). Consistent with the observation reported for the blaA knockout of $F$. tularensis subsp. novicida, we found that the $\Delta$ blaA1 mutants grew as well as wild-type cells on media containing ampicillin (Fig. 6).

We then made mutants with only a deletion of blaB, strains PM1556 (LVS $\Delta$ blaB1) and PM1580 (Schu $\Delta$ blaB1). These mutants had increased susceptibility to ampicillin and could be distinguished from wild-type or $\triangle$ blaA mutants on medium containing ampicillin at $50 \mu \mathrm{g} \mathrm{ml}^{-1}$ (Fig. 6). In addition, we constructed double mutants, strains PM1560 (LVS $\Delta$ blaA1 $\Delta$ blaB1) and PM1571 (Schu $\Delta$ blaA1 $\Delta$ blaB1). These mutants were comparable to the single $\triangle$ blaB1 mutants (Fig. 6). We confirmed allelic exchange in these mutants by Southern blotting as shown in Fig. 5 .

We then demonstrated that $b l a B^{+}$could be used as an additional selectable marker in the $\triangle$ blaA1 $\triangle$ blaB1 mutants. We cloned the wild-type $b l a B$ and its upstream region into the pMP529 shuttle vector to produce pMP615. This plasmid and the vector control were electroporated into the LVS and Schu double mutants, followed by dilution plating onto media containing either hygromycin at $200 \mu \mathrm{g} \mathrm{ml} \mathrm{m}^{-1}$ or

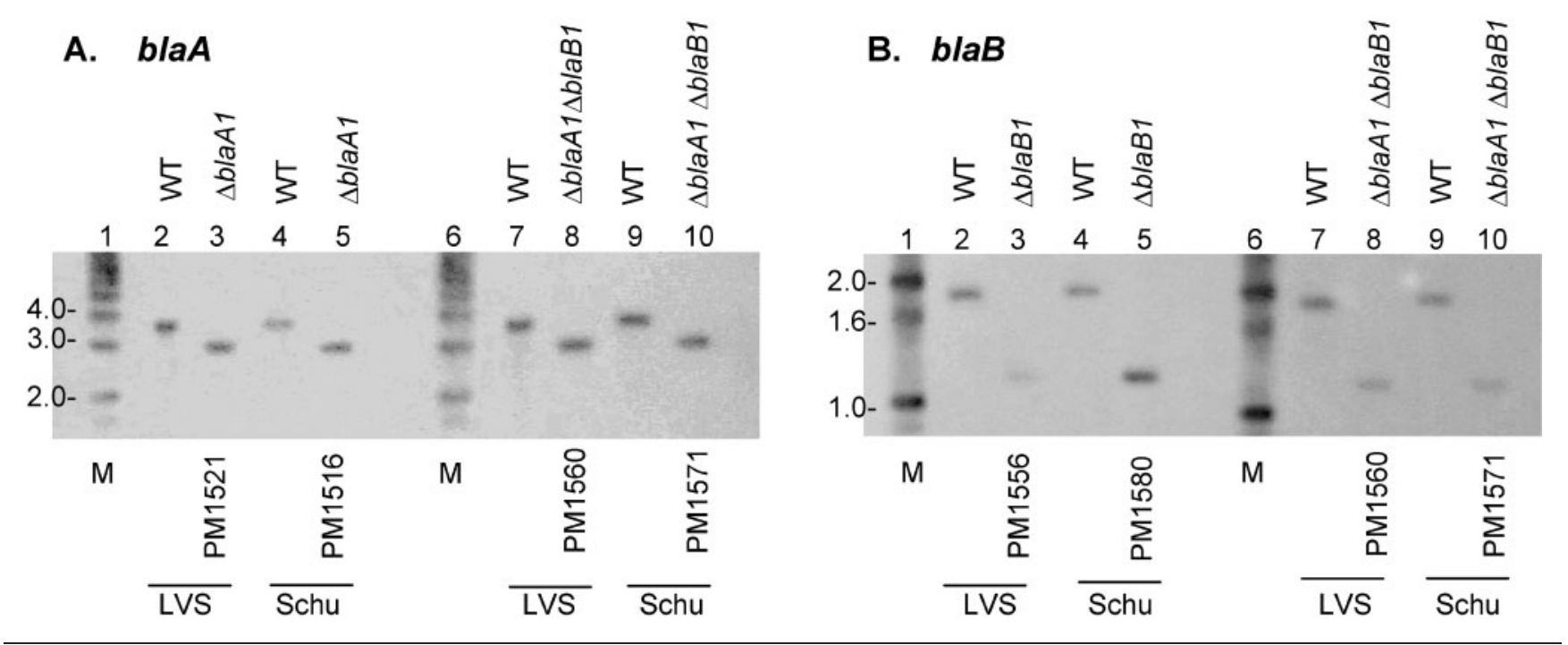

Fig. 5. Southern blots of genomic DNA from $\beta$-lactamase deletion mutants. (A) Genomic DNA of strains LVS and Schu digested with $\mathrm{Hpal}$ and probed with a $2 \cdot 3 \mathrm{~kb} P$ stl fragment from plasmid pMP552 encompassing the $\triangle b / a A 1$ allele. Lanes: 1 , 6, kb markers; 2, 7 and 4, 9, wild-type blaA (3.8 kb) LVS, Schu; 3, 5, $\Delta b l a A 1$ (3.0 kb) LVS, Schu mutants; 8, 10, $\Delta b / a A 1$ $(3.0 \mathrm{~kb})$ LVS, Schu double mutants. (B) Genomic DNA of strains LVS and Schu digested with Ndel and probed with a $1.0 \mathrm{~kb}$ Pstl fragment from plasmid pMP537 encompassing the $\triangle$ blaB1 allele. Lanes: 1, 6, kb markers; 2, 7 and 4, 9, wild-type blaB (1.9 kb) LVS, Schu; 3, 5, $\Delta$ blaB1 (1.2 kb) LVS, Schu mutants; 8, 10, $\Delta$ blaB1 (1.2 kb) LVS, Schu double mutants. 


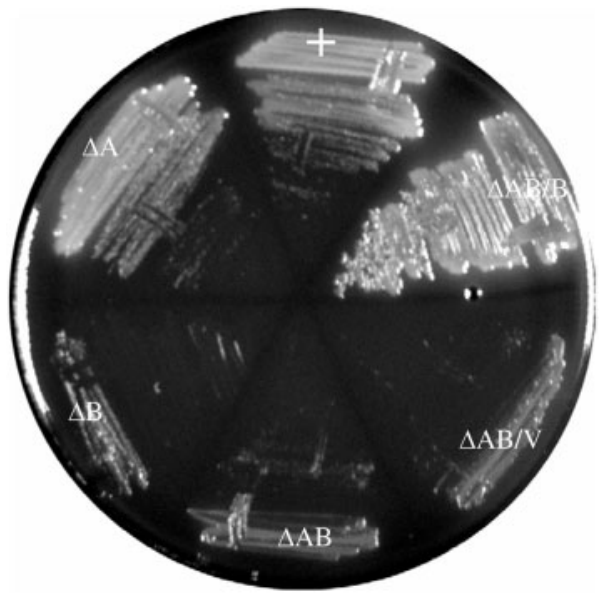

Fig. 6. Growth of LVS strains on $\mathrm{MMH}$ medium containing ampicillin at $50 \mu \mathrm{g} \mathrm{ml}^{-1}$. +, wild-type LVS; $\triangle \mathrm{A}, \Delta b / a A 1$ mutant $\mathrm{PM} 1521 ; \Delta \mathrm{B}, \Delta$ blaB1 mutant $\mathrm{PM} 1556 ; \triangle \mathrm{AB}, \Delta b / a A 1 \Delta b l a B 1$ mutant $\mathrm{PM} 1560 ; \triangle \mathrm{AB} / \mathrm{V}$, the $\triangle b l a A 1 \quad \Delta b l a B 1$ mutant vector control; $\triangle \mathrm{AB} / \mathrm{B}^{+}$, the double mutant complemented with gene $b / a B^{+}$in trans. Similar results were obtained with the corresponding Schu strains.

ampicillin at $50 \mu \mathrm{g} \mathrm{ml}^{-1}$. We obtained $\sim 10^{6}$ transformants per electroporation with both plating methods for both strains regardless of antibiotic, with pMP615 generating equivalent numbers of hygromycin- and ampicillin-resistant clones (data not shown). As expected, electroporations with pMP529 generated only hygromycin-resistant clones. Thus, blaB $^{+}$, carried on a plasmid in trans, complements the ampicillin phenotype and can be used for direct selection of ampicillin-resistant transformants in a $\triangle$ blaA1 $\triangle$ blaB1 background. The amount of $\mathrm{BlaB}$ enzyme expressed from the plasmid in the complemented strains appears to be higher than that produced by wild-type or $\Delta$ blaA1 cells, as indicated by the relatively better growth of the complemented strains on ampicillin plates (Fig. 6).

\section{DISCUSSION}

The plasmids described here were derived from the cryptic plasmid pFNL10 originally found in ' $F$. novicida-like strain F6168', a strain isolated from human blood in 1984 (Hollis et al., 1989; Pomerantsev et al., 2001a). This plasmid is $3990 \mathrm{bp}$ in size, carries six putative ORFs and is believed to replicate by the theta mode (Pomerantsev et al., 2001a). Putative functions of the ORFs have been inferred from sequence comparisons to known proteins and the phenotypes of deletion derivatives of other pFNL10-based shuttle vectors (Maier et al., 2004; Pavlov et al., 1996; Pomerantsev et al., 2001a, b). ORF1 is also known as repA and encodes the replication initiation protein, while ORF2 may be a helicase or endonuclease important for plasmid multimer resolution. The function of ORF3 is unknown but it has some similarity to recombinases and integrases, while ORF4 and ORF5 may constitute an addiction system important for maintenance of pFNL10 (Pomerantsev et al., 2001a, b). Addiction systems, or toxin-antitoxin systems, encode a long-lived toxic protein and a short-lived antitoxin protein. Loss of the two genes results in death or stasis of the cell by the toxic protein after the antitoxin is degraded by cellular proteases, as reviewed by Gerdes et al. (2005). The ORF5 gene product is similar to the Phd antitoxin encoded on bacteriophage P1 (part of the phd-doc addiction system of $\mathrm{P} 1$ ) and it is thought that the ORF4 gene product is the corresponding toxin, although its similarity to other toxic proteins, particularly Doc, is not very high (Pomerantsev et al., 2001a). The remaining ORF of pFNL10, ORFm, may encode a protein with a role in regulating the expression or the functions of ORF4 and ORF5, but this hypothesis is untested (Pomerantsev et al., 2001a).

Plasmid pFNL10 was previously used to create the first shuttle vector that could replicate in E. coli and F. tularensis. This vector, pFNL100, was made by fusing pFNL10 to the $E$. coli vector pBR328, and encodes resistance to tetracycline and chloramphenicol (Norqvist et al., 1996). A deletion derivative of this vector, pFNL200, which lacks the E. coli origin, was used in the development of the shuttle vector pKK202, which bears the E. coli p15A origin from pACYC184 (Norqvist et al., 1996). A more recent shuttle vector, pTOPO/FNL10, was made in much the same manner by fusing all of pFNL10 to an E. coli cloning vector bearing a kanamycin resistance marker (Maier et al., 2004). Both pFNL200 and pTOPO/FNL10 contained a segment of pFNL10 DNA that was ultimately unstable in $F$. tularensis and was spontaneously deleted from the vectors when passed through the organism. In the case of pFNL200, the deletion derivative, pOM1 (Pomerantsev et al., 2001a, b), lost part of ORF5, all of ORF4 and ORFm, while the deletion derivatives of pTOPO/FNL10 lost all of ORFm, all or most of ORF4, and in some cases (pFNLTP1), a part of ORF5 (Maier et al., 2004). In the construction of pFNL200, the cloning vector was inserted into ORF5, the putative antitoxin gene, while in the construction of pTOPO/FNL10, the cloning vector was inserted between ORFm and ORF3, which might have affected expression of ORFm or ORF4ORF5. These other researchers suggested that the deletion derivatives perhaps arose because the ORF4-ORF5 addiction system was probably dysregulated, with lethal consequences for F. tularensis.

In the construction of our first generation of shuttle vectors, we started with the minimal amount of DNA required for efficient replication in F. tularensis. However, these vectors were lost in the absence of selection, as demonstrated by the behaviour of pMP527. To rectify this, we inserted the ORF4-ORF5 region into our second generation of shuttle vectors. Our data from experiments with these vectors support the idea that these genes encode an addiction system because our second-generation plasmids are maintained in populations in the absence of selection.

The shuttle vector pFNLTP1, one of the deletion derivatives of pTOPO/FNL10 that contains repA, ORF2 and ORF3, is 
stable in the absence of selective pressure (Maier et al., 2004). Our stably maintained plasmids, pMP607 and pMP633, have repA, ORF2 and ORF4-ORF5 but lack ORF3. We think the maintenance of these two types of plasmids is the result of different mechanisms. Our original plasmids, pMP393, pMP527 and pMP529, have only repA and ORF2 and probably do not segregate as efficiently as pFNLTP1. We propose that the presence of the ORF4-ORF5 addiction system in our second-generation plasmids pMP607 and pMP633 does not increase the efficiency of plasmid segregation, but instead affects the maintenance of the plasmids in the population, as any daughter cell that lost the plasmid would be killed by the ORF4-encoded toxin. In contrast, the ORF3 gene present in pFNLTP1 might directly increase segregation efficiency by affecting plasmid partitioning, or it might play a role in copy number control. High-copy-number plasmids are less likely to be lost in daughter cells compared to low-copy-number plasmids.

It is possible that a plasmid bearing ORF3 is not well tolerated by strain Schu, as we did not obtain good transformation efficiencies with pFNLTP1 and Schu compared to our plasmids that lack ORF3. Furthermore, the Schu/ pFNLTP1 transformants did not grow well and had altered colony morphologies. This could be explained by a low tolerance of high-copy-number plasmids by Schu, if ORF3 has a role in copy-number control. Alternatively, the effects of pFNLTP1 on strain Schu could be related to the plasmid's kanamycin resistance marker $n p t$, which is different from the aphA-1 marker present in our vectors.

The tools described here can be further developed for new applications. We are redesigning the shuttle vectors to include the blaB promoter region and a multiple cloning site for use as expression vectors. The unstable shuttle vectors can be used for transient expression of site-specific recombinases for the removal of antibiotic cassettes or for gene essentiality tests. We anticipate that these tools will be useful to others in the field meeting the challenge of $F$. tularensis genetics.

\section{NOTE ADDED IN PROOF}

While this paper was in review, another group (Bina, X. R., Wang, C, Miller, M. A. \& Bina, J. E. 2006 Arch Microbiol 186, 219-228) reported on the expression of the F. tularensis $\beta$ lactamase genes in $E$. coli and their data support our conclusions here that only the blaB gene contributes to $\beta$ lactamase resistance in F. tularensis. Another group (Qin, A. \& Mann, B. J. 2006 BMC Microbiol 6, 69) reported transposon mutagenesis of F. tularensis strain SchuS4 with the EZ :: TN system using rifampicin resistance.

\section{ACKNOWLEDGEMENTS}

This work was supported by grants AI55551 and GM064133 from the National Institutes of Health. M. S. P. thanks Tom Zahrt for pFNLTP1 and discussions about Francisella genetics, Fran Nano for generously supplying pFNL10 DNA, Jorge Benach for LVS, Karen Elkins for Utah 112, Michael Schriefer for Schu, and Miriam Braunstein and members of the Pavelka lab for reviewing this manuscript. We also wish to acknowledge Matthew Reader, a participant in the University of Rochester's Summer Scholar Program, for the construction of some of the plasmids used in this study.

\section{REFERENCES}

Ausubel, F. M., Brent, R., Kingston, R. E., Moore, D. D., Seidman, J. G., Smith, J. A. \& Struhl, K. (1987). Current Protocols in Molecular Biology. New York: Greene Publishing Associates and Wiley-Interscience.

Baker, C. N., Hollis, D. G. \& Thornsberry, C. (1985). Antimicrobial susceptibility testing of Francisella tularensis with a modified Mueller-Hinton broth. J Clin Microbiol 22, 212-215.

Cowley, S. C., Gray, C. J. \& Nano, F. E. (2000). Isolation and characterization of Francisella novicida mutants defective in lipopolysaccharide biosynthesis. FEMS Microbiol Lett 182, 63-67.

Dennis, D. T., Inglesby, T. V., Henderson, D. A. \& 15 other authors (2001). Tularemia as a biological weapon: medical and public health management. JAMA 285, 2763-2773.

Derbyshire, K. M., Takacs, C. \& Huang, J. (2000). Using the EZ:: TN transposome for transposon mutagenesis in Mycobacterium smegmatis. Epicentre Forum 7, 1-4.

Eigelsbach, H. T. \& Downs, C. M. (1961). Prophylactic effectiveness of live and killed tularemia vaccines. I. Production of vaccine and evaluation in the white mouse and guinea pig. I Immunol 87, 415-425.

Ericsson, M., Golovliov, I., Sandstrom, G., Tarnvik, A. \& Sjostedt, A. (1997). Characterization of the nucleotide sequence of the groE operon encoding heat shock proteins chaperone-60 and -10 of Francisella tularensis and determination of the T-cell response to the proteins in individuals vaccinated with $F$. tularensis. Infect Immun 65, 1824-1829.

Flores, A. R., Parsons, L. M. \& Pavelka, M. S., Jr (2005a). Characterization of novel Mycobacterium tuberculosis and Mycobacterium smegmatis mutants hypersusceptible to beta-lactam antibiotics. J Bacteriol 187, 1892-1900.

Flores, A. R., Parsons, L. M. \& Pavelka, M. S., Jr (2005b). Genetic analysis of the beta-lactamases of Mycobacterium tuberculosis and Mycobacterium smegmatis and susceptibility to beta-lactam antibiotics. Microbiology 151, 521-532.

Garcia Del Blanco, N., Dobson, M. E., Vela, A. I. \& 7 other authors (2002). Genotyping of Francisella tularensis strains by pulsed-field gel electrophoresis, amplified fragment length polymorphism fingerprinting, and 16S rRNA gene sequencing. J Clin Microbiol 40, 2964-2972.

Gerdes, K., Christensen, S. K. \& Lobner-Olesen, A. (2005). Prokaryotic toxin-antitoxin stress response loci. Nat Rev Microbiol 3, 371-382.

Golovliov, I., Sjostedt, A., Mokrievich, A. \& Pavlov, V. (2003). A method for allelic replacement in Francisella tularensis. FEMS Microbiol Lett 222, 273-280.

Goryshin, I. Y., Jendrisak, J., Hoffman, L. M., Meis, R. \& Reznikoff, W. S. (2000). Insertional transposon mutagenesis by electroporation of released Tn5 transposition complexes. Nat Biotechnol 18, 97-100.

Hollis, D. G., Weaver, R. E., Steigerwalt, A. G., Wenger, J. D., Moss, C. W. \& Brenner, D. J. (1989). Francisella philomiragia comb. nov. (formerly Yersinia philomiragia) and Francisella tularensis biogroup novicida (formerly Francisella novicida) associated with human disease. J Clin Microbiol 27, 1601-1608. 
Johansson, A., Goransson, I., Larsson, P. \& Sjostedt, A. (2001). Extensive allelic variation among Francisella tularensis strains in a shortsequence tandem repeat region. J Clin Microbiol 39, 3140-3146.

Johansson, A., Farlow, J., Larsson, P. \& 8 other authors (2004). Worldwide genetic relationships among Francisella tularensis isolates determined by multiple-locus variable-number tandem repeat analysis. J Bacteriol 186, 5808-5818.

Kawula, T. H., Hall, J. D., Fuller, J. R. \& Craven, R. R. (2004). Use of transposon-transposase complexes to create stable insertion mutant strains of Francisella tularensis LVS. Appl Environ Microbiol 70, 6901-6904.

Lauriano, C. M., Barker, J. R., Nano, F. E., Arulanandam, B. P. \& Klose, K. E. (2003). Allelic exchange in Francisella tularensis using PCR products. FEMS Microbiol Lett 229, 195-202.

Leboul, J. \& Davies, J. (1982). Enzymatic modification of hygromycin B in Streptomyces hygroscopicus. J Antibiot 35, 527-528.

Maier, T. M., Havig, A., Casey, M., Nano, F. E., Frank, D. W. \& Zahrt, T. C. (2004). Construction and characterization of a highly efficient Francisella shuttle plasmid. Appl Environ Microbiol 70, 7511-7519.

Maier, T. M., Pechous, R., Casey, M., Zahrt, T. C. \& Frank, D. W. (2006). In vivo Himar1-based transposon mutagenesis of Francisella tularensis. Appl Environ Microbiol 72, 1878-1885.

Malpartida, F., Zalacain, M., Jimenez, A. \& Davies, J. (1983). Molecular cloning and expression in Streptomyces lividans of a hygromycin B phosphotransferase gene from Streptomyces hygroscopicus. Biochem Biophys Res Commun 117, 6-12.

Nano, F. E., Zhang, N., Cowley, S. C. \& 8 other authors (2004). A Francisella tularensis pathogenicity island required for intramacrophage growth. J Bacteriol 186, 6430-6436.

Norqvist, A., Kuoppa, K. \& Sandstrom, G. (1996). Construction of a shuttle vector for use in Francisella tularensis. FEMS Immunol Med Microbiol 13, 257-260.
Pavelka, M. S., Jr \& Jacobs, W. R., Jr (1999). Comparison of the construction of unmarked deletion mutations in Mycobacterium smegmatis, Mycobacterium bovis bacillus Calmette-Guerin, and Mycobacterium tuberculosis $\mathrm{H} 37 \mathrm{Rv}$ by allelic exchange. J Bacteriol 181, 4780-4789.

Pavlov, V. M., Mokrievich, A. N. \& Volkovoy, K. (1996). Cryptic plasmid pFNL10 from Francisella novicida-like F6168: the base of plasmid vectors for Francisella tularensis. FEMS Immunol Med Microbiol 13, 253-256.

Pomerantsev, A. P., Golovliov, I. R., Ohara, Y., Mokrievich, A. N., Obuchi, M., Norqvist, A., Kuoppa, K. \& Pavlov, V. M. (2001a). Genetic organization of the Francisella plasmid pFNL10. Plasmid 46, 210-222.

Pomerantsev, A. P., Obuchi, M. \& Ohara, Y. (2001b). Nucleotide sequence, structural organization, and functional characterization of the small recombinant plasmid pOM1 that is specific for Francisella tularensis. Plasmid 46, 86-94.

Shaw, K. J., Rather, P. N., Hare, R. S. \& Miller, G. H. (1993). Molecular genetics of aminoglycoside resistance genes and familial relationships of the aminoglycoside-modifying enzymes. Microbiol Rev 57, 138-163.

Sjostedt, A., Tarnvik, A. \& Sandstrom, G. (1996). Francisella tularensis: host-parasite interaction. FEMS Immunol Med Microbiol 13, 181-184.

Stover, C. K., de la Cruz, V. F., Fuerst, T. R. \& 28 other authors (1991). New use of BCG for recombinant vaccines. Nature 351, 456-460.

Titball, R. W., Johansson, A. \& Forsman, M. (2003). Will the enigma of Francisella tularensis virulence soon be solved? Trends Microbiol 11, 118-123.

Twine, S., Bystrom, M., Chen, W. \& 9 other authors (2005). A mutant of Francisella tularensis strain SCHU S4 lacking the ability to express a 58-kilodalton protein is attenuated for virulence and is an effective live vaccine. Infect Immun 73, 8345-8352. 